\title{
AVALIAÇÃO DE DIFERENTES CULTIVARES DE SOJA NO MUNICÍPIO DE SÃO JOSÉ DO XINGU-MT
}

\section{VALUATION OF DIFFERENT SOYBEAN CROP IN THE CITY OF SÃO JOSÉ DO XINGU-MT}

\author{
Jeisi Mara Rasia'; José Antonio do Vale Sant'Ana ${ }^{2}$
}

DOI: $\underline{\text { https://doi.org/10.31692/978-65-991061-7-0.121-132 }}$

\begin{abstract}
RESUMO
A obtenção de altos rendimentos na produção da cultura da soja (Glycine max (L.) Merrill) está relacionada com a interação entre planta, ambiente e manejo. $\mathrm{O}$ correto posicionamento de cultivares de soja é fundamental para o sucesso dos produtos disponíveis no mercado e a otimização dos sistemas agronômicos em que estão inseridos, atua-se diretamente no desenvolvimento da cultura, e o efeito será a produtividade. Com trabalhos realizados em regiões especificas assegura-se que a variedade possa expressar a melhor performance genética possível dentro da condição ambiental em que foi implantada. Objetivou-se com este trabalho avaliar através de caracteres agronômicos o desempenho de diferentes cultivares de soja no município de São José do Xingu-MT, durante a safra 2017/2018. O delineamento experimental foi realizado em blocos ao acaso nas dimensões $18 \mathrm{~m} \times 12 \mathrm{~m}$, contendo três blocos, cada bloco com seis parcelas (tratamento) de $2 \mathrm{~m}$ x $5 \mathrm{~m}$ cada, cada parcela com quatro linhas e espaçamento entre linhas de $0,5 \mathrm{~m}$. Foram avaliados os caracteres agronômicos: germinação, altura de inserção da primeira vagem, altura de planta na maturação, quantidade de vagem por planta, quantidade de grãos por planta, e produtividade em sacas por hectare (sc/ha). Os dados foram analisados através da análise de variância (teste $\mathrm{F}$ ), e as médias comparadas pelo teste de Tukey ao nível de 5\% de probabilidade, utilizando o programa estatístico SISVAR. De forma geral as cultivares não se diferenciaram entre si e obtiveram resultados positivos, com exceção da BRS 7380RR que não apresentou resultado satisfatório conforme a média de produção atingida na região do experimento para safra 2017/2018.
\end{abstract}

Palavras-Chave: Desempenho, Glycine max, Produtividade.

\begin{abstract}
Obtaining high yields in the soybean crop (Glycine max (L.) Merrill) is related to the interaction between plant, environment and management. The correct positioning of soybean cultivars is fundamental for the success of the products available in the market and the optimization of the agronomic systems in which they are inserted, it acts directly in the development of the crop, and the effect will be the productivity. With works carried out in specific regions ensures that the variety can express the best genetic performance possible within the environmental condition in which it was implanted. The objective of this work was to evaluate the performance of different soybean crops in the city of São José do Xingu-MT, during the 2017/2018 harvest, using agronomic characters. The experimental design was realized in randomized blocks, in dimensions $18 \times 12$ meters, containing 3 blocks, each block of six parcels (treatment) of 2 x 5 meters each, parcel with four lines and spacing among lines of 0,5 meters. The agronomic characters evaluated were: germination, stature of insertion of the first pod, stature of plant in maturity, amount of pod for plant, amount of grains per plant,

\footnotetext{
${ }^{1}$ Bacharelado em Agronomia, Instituto Federal de Educação, Ciência e Tecnologia de Mato Grosso-Campus Confresa, jeisiagronoma@gmail.com

2 Doutor em Recursos Hídricos e professor, Instituto Federal de Educação, Ciência e Tecnologia de Mato Grosso-Campus Confresa, jose.santana@cfs.ifmt.edu.br
} 
and productivity in sacks per hectare (sc/ha). The data were analyzed through analysis of variance ( $\mathrm{F}$ test), and the means were compared by Tukey test at the $5 \%$ probability level, using the statistical program SISVAR. In general, the crops did not differentiate from one another and obtained positive results, with the exception of BRS 7380RR, that did not demonstrate satisfactory results, according to the average production achieved in the experiment region for the 2017/2018 harvest.

Keywords: Performance, Glycine max, Productivity.

\section{INTRODUÇÃO}

A soja (Glycine $\max (\mathrm{L}$.$) Merrill) tem como centro de origem o continente asiático,$ mais precisamente, a região correspondente à China Antiga, sendo essa leguminosa a base alimentar do povo chinês há mais de 5.000 anos (CÂMARA, 1998).

No Brasil a boa rentabilidade da cultura estimularam os produtores a cultivá-la, sendo que o plantio nacional apresentou aumento na área de 3,5\% e desde a safra de 2006/07 o país ganhou 14,4 milhões de hectares novos de soja, se tornando a cultura protagonista no aumento da área que atualmente corresponde a $57 \%$ da área total semeada com grãos, saindo de $33.909,4$ mil hectares na safra 2016/17 para 35.089,8 mil novos hectares na atual 2017/18 (CONAB, 2013; CONAB, 2018).

Para a produção brasileira de soja safra 2018/19 há uma estimativa do Departamento de Agricultura dos Estados Unidos da América (USDA) de um recorde de 120,5 milhões de toneladas, segundo este mesmo departamento, para a área colhida também haverá um recorde de 37,5 milhões de hectares continuando a se expandir como vem ocorrendo nos últimos 10 anos, sendo que 2018/19 deverá apresentar o maior aumento anual em cinco anos, a produção dos EUA deve chegar a 116,48 milhões de toneladas de soja (safra de 2018/19), menor que a produção brasileira em 2018 (já colhida) de 117 milhões de toneladas, ultrapassando assim os EUA em produção e se tornando pela primeira vez o maior produtor mundial de soja (USDA, 2018).

A obtenção de altos rendimentos na cultura da soja (Glycine max (L.) Merrill) está relacionada com a interação entre planta, ambiente e manejo (FREITAS et al., 2010). De acordo com Borém e Miranda (2009) o melhoramento genético das plantas busca esta interação, desenvolvendo genótipos resistentes ou tolerantes às pragas, doenças e estresses climáticos, adaptados a regiões específicas. Conforme Peixoto et al. (2000), as características quantitativas como componentes do rendimento (número de vagens por planta, número de grãos por vagem e massa dos grãos), altura de planta, duração do ciclo e produtividade, são as mais importantes na escolha das cultivares sendo as mais influenciadas pelo manejo.

Tendo em vista as escassas informações e dados literários sobre cultivares de soja 
adaptadas para a região Araguaia-Xingu, objetivou-se com este trabalho avaliar através de caracteres agronômicos o desempenho de diferentes cultivares de soja no município de São José do Xingu-MT, durante a safra 2017/2018.

\section{FUNDAMENTAÇÃO TEÓRICA}

Para Almeida et al. (2016) a escolha correta das cultivares mais adaptadas às condições locais é de fundamental importância visando à expressão do máximo potencial produtivo e maior custo/benefício da lavoura. Altos rendimentos somente são obtidos quando as condições ambientais são favoráveis em todos os estágios de crescimento da soja (BALBINOT JUNIOR, 2018).

A análise da fertilidade do solo também é de grande importância para a soja, pois a cultura possui grande exigência em macro nutrientes $(\mathrm{N}, \mathrm{P}, \mathrm{K}, \mathrm{Ca}, \mathrm{S}$ e $\mathrm{Mg})$ e micro nutrientes (Fe, Mn, $\mathrm{Cu}, \mathrm{Zn}, \mathrm{B}, \mathrm{Mo}$, e CL), e seus teores ideais podem ser corrigidos antes da implantação da cultura. Esses elementos minerais estão diretamente ligados aos processos metabólicos da planta, sendo seus benefícios indiscutíveis em relação ao seu desenvolvimento e crescimento, consequentemente proporcionando boas condições para uma alta produtividade (PROCHNOW e ROSSI, 2009).

A obtenção de elevadas produtividades requer também adequadas populações de plantas, dependentes da região, da época de semeadura e da cultivar, pela maximização dos componentes da produção. Até a década de 1980, era comum a produção de soja com 400 mil plantas/ha ou superior. Evoluções, como a elevação da qualidade das sementes produzidas no país e a recomendação de novas cultivares, possibilitaram a redação da população de plantas para cerca de 230 mil plantas/ha. (ROSSI, 2017).

Freitas (2011), afirma que boas condições físicas dos solos facilitam as operações com máquinas agrícolas, e o regime pluviométrico altamente favorável e o baixo valor da terra, fazendo o bioma Cerrado no Brasil Central ser responsável por quase 50\% da produção nacional de soja. Segundo Fernández (2007), a história da soja em Mato Grosso é recente e coincide com a trajetória da migração dos colonos gaúchos, a partir dos anos de 1960, incentivada pelas políticas de colonização, de infraestrutura, de créditos subsidiados e de pesquisa agropecuária. Portanto, a "expansão" das lavouras não é retratada apenas pelo

crescimento da área plantada, mas por um movimento mais amplo de produção e transformação de territórios, de formas de ocupação da terra e de estruturação de relações sociais.

De acordo com o boletim técnico de Decicino (2015) o correto posicionamento de 
cultivares de soja é fundamental para o sucesso dos produtos disponíveis no mercado e a otimização dos sistemas agronômicos em que estão inseridos, tanto que empresas de germoplasma que atuam no mercado de sementes de soja exigem o posicionamento adequado dos materiais por regiões, fator para a competitividade comercial e consolidação das cultivares no mercado. Quando se posiciona uma cultivar de soja, atua-se diretamente no desenvolvimento da cultura, e o efeito será a produtividade.

O posicionamento de cultivares de soja depende de diversos fatores sobre os quais atuamos diretamente, como níveis de fertilidade, época de semeadura, distribuição espacial e fatores genéticos. O posicionamento engloba todos esses tópicos; assim, o conhecimento e o correto uso dessas variáveis são importantes para alcançar o melhor resultado diante de um fator que não pode ser controlado: o clima. Assim, com trabalhos realizados em regiões especificas assegura-se que a variedade possa expressar a melhor performance genética possível dentro da condição ambiental em que foi implantada, e também podendo descartar cultivares não adaptadas aquele local especifico (DECICINO, 2015).

\section{METODOLOGIA}

O experimento foi conduzido na Fazenda Bang-Bang, localizada a $10^{\circ} 48^{\prime} 40,9^{\prime \prime} \mathrm{S}$, $52^{\circ} 43^{\prime} 41,0$ ” W município de São José do Xingu-MT, na região Nordeste do Estado, com altitude de $335 \mathrm{~m}$ e solo de textura franco arenosa classificado de acordo com as características descritas na Tabela 1, em condições de campo e área de segundo ano de plantio, na safra 2017/18 (VALLE et al., 2000; IBGE, 2009; DOMINGUES, 2010; BRASIL, 2018).

O delineamento experimental foi realizado em blocos ao acaso, nas dimensões $18 \mathrm{~m} x$ $12 \mathrm{~m}$ contendo três blocos, cada bloco com seis parcelas (tratamento) de $2 \mathrm{~m}$ x $5 \mathrm{~m}$ cada , cada parcela contendo quatro linhas com espaçamento entre linhas de $0,5 \mathrm{~m}$. As linhas de plantio foram delimitadas através de plantadeira mecanizada, e o plantio realizado manualmente no dia 14 de novembro de 2017, com 20 sementes por metro linear, sendo realizado desbaste aos 17 DAP (dias após o plantio) de acordo com as recomendações de cada cultivar. Os dados foram avaliados a partir da coleta de 10 plantas nas linhas centrais das parcelas, desconsiderando as laterais para efeito de bordadura.

Tabela 1. Análise de solo da área experimental (profundidade da amostra 10-20 cm). Fonte: Própia.

\begin{tabular}{cc}
\hline CARACTERÍSTICAS & RESULTADOS \\
\hline $\mathrm{pH} \mathrm{CaCl}{ }_{2}$ & 5,10 \\
$\mathrm{Ca}^{2+}\left(\mathrm{cmolc}_{\mathrm{dm}}{ }^{3}\right)$ & 1,90
\end{tabular}




$\begin{array}{cc}\mathrm{Mg}^{2+}\left(\mathrm{cmolc} / \mathrm{dm}^{3}\right) & 0,60 \\ \mathrm{~K}^{+} \text {disponível }\left(\mathrm{mg} / \mathrm{dm}^{3}\right) & 0,12 \\ \mathrm{P} \text { mel }\left(\mathrm{mg} / \mathrm{dm}^{3}\right) & 12,30 \\ \mathrm{H}^{+} \mathrm{Al}(\mathrm{m} \mathrm{E} / 100 \mathrm{cc}) & 2,60 \\ \mathrm{M} \mathrm{O}(\mathrm{g} / \mathrm{kg}) & 25,90 \\ \text { Areia }(\%) & 78 \\ \text { Silte }(\%) & 5 \\ \text { Argila }(\%) & 17\end{array}$

As seis cultivares de soja (tratamentos) utilizadas foram: Agro Norte 73017 RR, Pioneer 96 Y90 RR, BRS 7780 IPRO, BRS 7680 RR, BRS 7380 RR, e BRS 8381.

Algumas das cultivares utilizadas são transgênicas, e apresentam a tecnologia Roundup Ready (resistência ao glifosato) e apenas uma convencional, sendo elas:

- Pioneer 96Y90 RR, resistente ao nematoide de cisto (Heterodera glycines Ichinohe), de alto potencial produtivo, e habito de crescimento indeterminado, tolerante ao acamamento, baixo fator de reprodução de nematoides Meloydogine incógnita e $M$. javanica, seu ciclo é precoce (SOJA, 2018);

- Agro Norte 73017 RR, com crescimento semi-determinado, moderadamente tolerante a seca, variedade de ciclo precoce, altura de plantas em torno de $86 \mathrm{~cm}$, e inserção de primeira vagem aos 4,7cm (AGRONORTE, 2018);

- BRS 7380 RR com resistência as raças 3, 4, 6, 9, 10, 14 do nematoide de cisto Heteroda glycines Ichinohe, e os de galha Meloidogyne incógnita e M. javanica, tipo de crescimento indeterminado, e sendo lançada no ano de 2015;

- BRS 7680 RR, de ciclo precoce, resistência aos nematoides de galhas Meloidogyne incógnita e M. javanica, tolerância ao vírus da necrose da haste, planta de porte médio e crescimento indeterminado, sendo lançada em 2016;

- BRS 7780 IPRO que apresenta a tecnologia INTACTA RR2 PRO com proteção contra as principais lagartas da soja (lagarta da soja Anticarsia gemmatalis; lagarta falsa medideira Chrysodexis includens; broca das axilas Epinotia aporema; e lagarta das maçãs Heliothis virescens) possui crescimento semi-determinado, ciclo precoce, e resistência ao nematoide de galha Meloidogyne incógnita (FUNDAÇÃO, 2018).

- Como cultivar convencional, se utilizou a BRS 8381, que apresenta tipo de crescimento ereto e semi-determinado, ciclo precoce, medianamente resistente ao nematoide de galha Meloidogyne javanica (FUNDAÇÃO, 2018).

Antes do plantio destas cultivares realizou-se a dessecação da área com glifosato, na qual estava implantada a cultura do milheto exclusivamente para cobertura de palhada; também se realizou aplicação de Borotop, e Starter manganês. O tratamento de semente foi 
realizado a base de Dermacor, Protreat, e Comor.

O controle de plantas daninhas da área foi realizado através de capina manual, já a adubação, e o controle de pragas e doenças foram realizados conforme a necessidade e manejo da fazenda para o talhão que incluía o experimento, recomendados pelo técnico responsável. Aos 36 DAP fez-se a primeira aplicação de fungicida Fox e Engeo, e aos 57 DAP a segunda aplicação com fungicida Fox e Connect, e aos 67 DAP aplicação de inseticida e fungicida utilizando Galil, e aos 72 DAP a quarta aplicação de fungicida com Horos e Acefato. Segundo os registros da fazenda, a precipitação pluviométrica no período foi de $1.650 \mathrm{~mm}$.

Foram avaliados os caracteres agronômicos germinação, realizada aos 17 DAP, aos 101 DAP em estágio R8 (maturação plena) se avaliou altura de inserção da primeira vagem, altura de planta, quantidade de vagem por planta, quantidade de grãos por planta, e produtividade em sacas por hectare (sc/ha), todas as características foram avaliadas em dez plantas por parcela selecionadas ao acaso nas duas linhas centrais.

Os dados foram analisados através da análise de variância (teste F), e as médias comparadas pelo teste de Tukey ao nível de 5\% de probabilidade (Pimentel e Garcia, 2002), utilizando o programa estatístico SISVAR (FERREIRA, 2000).

\section{RESULTADOS E DISCUSSÃO}

Houve diferença significativa entre as cultivares para as variáveis germinação, altura de plantas na maturação, e a altura de inserção de primeira vagem (Tabela 2) ao nível de 5\% de probabilidade segundo o teste $\mathrm{F}(\mathrm{P}<0,05)$.

Torres et al. (2014) ao avaliar estes mesmos caracteres em soja verificou diferenças significativas $(\mathrm{p}<0,05)$ para altura de planta e altura de inserção de primeira vagem. Rossi et al. (2017) não constatou diferenças na contagem da germinação e na porcentagem de germinação por influência de fatores agronômicos.

Tabela 2. Resumo da análise de variância para a germinação (GER), altura de inserção de primeira vagem (IPV), e altura de planta na maturidade (APM), sendo o grau de liberdade representado por (GL) e coeficiente de variância $(\mathrm{CV})$. Fonte: Própria.

\begin{tabular}{ccccc}
\hline \multirow{2}{*}{ Causa da variação } & \multirow{2}{*}{ GL } & \multicolumn{3}{c}{ Quadrados médios } \\
\cline { 3 - 5 } & & GER & IPV & APM \\
\hline CULTIVARES & 5 & $106,71^{*}$ & $28,29 *$ & $375,06^{*}$ \\
BLOCOS & 2 & 12,42 & 13,54 & 353,20 \\
Erro & 10 & 13,54 & 5,50 & 61,95 \\
\hline Média: & & 89,22 & 15,58 & 91,96 \\
CV $(\%):$ & & 4,12 & 15,06 & 8,56 \\
\hline
\end{tabular}

*significativo a $5 \% \mathrm{e}^{\mathrm{ns}}$ não significativo respectivamente pelo teste $\mathrm{F}$ a $\mathrm{p}<0,05$. 
Segundo verifica-se em Rezende e Carvalho (2007), Garcia et al. (2007), Heiffig e Câmara (2006), todas as cultivares (Tabela 3) obtiveram altura de inserção de primeira vagem satisfatórias de 10 a $12 \mathrm{~cm}$, porém apenas a Agro Norte 73017 RR, BRS 7680 RR, BRS 7380RR e BRS 8381 não diferenciaram entre si variando entre 13,56 cm e 20,03 cm. Heiffig e Câmara (2006) afirmam que nessa altura as cultivares se tornam adequadas à colheita mecanizada realizada por colhedoras mais modernas que podem efetuar a colheita com plantas apresentando altura de inserção de primeira vagem em torno de $10 \mathrm{~cm}$.

Tabela 3. Valores médios das variáveis, germinação (GER) em \%, inserção de primeira vagem (IPV), e altura de plantas na maturação (APM) em cm. Fonte: Própria.

\begin{tabular}{|c|c|c|c|}
\hline CULTIVARES & $\begin{array}{c}\text { GER } \\
(\%)\end{array}$ & $\begin{array}{l}\text { IPV } \\
(\mathrm{cm})\end{array}$ & $\begin{array}{c}\mathbf{A P M} \\
(\mathrm{cm})\end{array}$ \\
\hline AGRO NORTE 73017 RR & $81,66 \mathrm{c}$ & $13,56 \mathrm{ab}$ & $93,46 \mathrm{abc}$ \\
\hline PIONEER 96Y90 RR & $93,00 \mathrm{ab}$ & $12,53 \mathrm{~b}$ & $76,18 \mathrm{c}$ \\
\hline BRS 7380 RR & $95,91 \mathrm{a}$ & $17,38 a b$ & $99,15 \mathrm{ab}$ \\
\hline BRS7680 RR & $86,50 \mathrm{abc}$ & $20,03 \mathrm{a}$ & $103,43 \mathrm{a}$ \\
\hline BRS 7780 IPRO & $83,91 \mathrm{bc}$ & $12,75 \mathrm{~b}$ & $80,26 \mathrm{bc}$ \\
\hline BRS 8381 & $94,33 \mathrm{ab}$ & $17,23 \mathrm{ab}$ & $99,30 \mathrm{ab}$ \\
\hline
\end{tabular}

*As médias seguidas da mesma letra na coluna não diferem entre si, pelo teste de Tukey a 5\% de probabilidade.

Os valores médios de germinação apresentam-se acima de $80 \%$, em que a cultivar Agro Norte 73017RR apresentou o pior desempenho com 81,66\%, e a BRS7380RR apresentou melhor índice de germinação com 95,91\%, sendo que a mesma não se diferenciou das cultivares Pioneer 96Y90 RR, BRS 8381 e BRS 7680 RR (Tabela 3).

Em relação ao caractere altura de plantas, destacaram-se as cultivares Agro Norte 73017 RR, BRS 7380 RR, BRS 7680RR e BRS 8381 que obtiveram maiores alturas de planta, quando comparadas às cultivares Pioneer 96Y90 RR e BRS 7780 IPRO que apresentaram os menores valores, e não se diferenciaram entre si estatisticamente (Tabela 3). No entanto, de maneira geral todos esses valores estão de acordo com os padrões aceitáveis na literatura que cita alturas de plantas compreendidas entre 50 e $120 \mathrm{~cm}$ (REZENDE e CARVALHO 2007; e GARCIA et al., 2007).

Verificamos através da Tabela 3, que as cultivares BRS 7780IPRO e BRS 7380RR apresentaram maiores alturas de planta na maturação para este experimento na cidade de São José do Xingu do que para as alturas verificadas por Ramos et al. (2017) na cidade de SinopMT, onde a cultivar BRS 7780IPRO apresentou $45 \mathrm{~cm}$ e a BRS $7380 \mathrm{RR}$ obteve $52 \mathrm{~cm}$ de altura média de planta na maturação.

Podemos observar na Tabela 4, que houve diferença significativa para a produção de grãos e não houve para quantidade de grãos por planta e quantidade de vagens por planta. Possivelmente não ocorreu significância entre a quantidade de grãos e vagens por planta 
devido ao alto valor de coeficiente de variação, $\mathrm{CV}=23,23 \%$ e $\mathrm{CV}=23,26 \%$, respectivamente, sendo que para cultivares diferentes costumam ocorrer variações de peso e tamanho de grãos.

Nota-se que na cidade de São José do Xingu-MT, as cultivares utilizadas neste experimento apresentam para quantidade de vagem variação entre 31,90 e 47,33 vagens por planta, e quantidade de 77,26 e 115,06 grãos por planta (Tabela 4).

Tabela 4. Análise de variância do quadrado médio para a quantidade de vagens por planta (QVP), quantidade de grãos por planta (QGP) e produtividade de grãos (PG), sendo o grau de liberdade representado por (GL) e coeficiente de variância (CV). Fonte: Própria.

\begin{tabular}{ccccc}
\hline \multirow{2}{*}{ Causa da variação } & \multirow{2}{*}{ GL } & \multicolumn{3}{c}{ Quadrados médios } \\
\cline { 3 - 5 } & 5 & QVP & QGP & PG \\
\hline CULTIVARES & 2 & $37,61^{\text {ns }}$ & $563,56^{\text {ns }}$ & $139,17^{*}$ \\
BLOCOS & 10 & 93,53 & 1668,02 & 1,41 \\
Erro & & 53,20 & 53,34 & 4,68 \\
\hline Média: & & 41,50 & 99,60 & 64,39 \\
CV $(\%):$ & 23,26 & 23,23 & 3,36 \\
\hline
\end{tabular}

*significativo a $5 \% \mathrm{e}^{\mathrm{ns}}$ não significativo respectivamente pelo teste $\mathrm{F}$ a $\mathrm{p}<0,05$.

A cultivar BRS 7680RR (Tabela 5) apresentou os menores valores médios para a produtividade de grãos $(51,06 \mathrm{sc} / \mathrm{ha})$, quando comparada com as demais cultivares que não diferenciaram-se entre si e apresentaram valores entre 64,64 e 69,31 sc/ha. Para a safra 2017/18, segundo informações obtidas com técnicos da região, a produtividade média alcançada pelos produtores foi de aproximadamente $60 \mathrm{sc} / \mathrm{ha}$, demonstrando que as cultivares testadas apresentaram resultados satisfatórios para a região, podendo ser recomendadas para o cultivo, exceto a cultivar BRS 7680RR.

Tabela 5. Valores médios das variáveis, quantidade de vagens por planta (QVP), quantidade de grãos por planta (QGP), e produtividade de grãos (PG) em sacas por hectare. Fonte: Própria.

\begin{tabular}{cccc}
\hline CULTIVARES & $\begin{array}{c}\text { QVP } \\
\text { (Un.) }\end{array}$ & $\begin{array}{c}\text { QGP } \\
\text { (Un.) }\end{array}$ & $\begin{array}{c}\text { PG } \\
(\mathrm{sc} / \mathrm{ha})\end{array}$ \\
\hline AGRO NORTE 73017 RR & $42,96 \mathrm{a}$ & $92,90 \mathrm{a}$ & $64,64 \mathrm{a}$ \\
PIONEER 96Y90 RR & $31,90 \mathrm{a}$ & $77,26 \mathrm{a}$ & $68,21 \mathrm{a}$ \\
BRS 7380 RR & $39,06 \mathrm{a}$ & $97,53 \mathrm{a}$ & $68,31 \mathrm{a}$ \\
BRS7680 RR & $47,33 \mathrm{a}$ & $115,06 \mathrm{a}$ & $51,06 \mathrm{~b}$ \\
BRS 7780 IPRO & $43,43 \mathrm{a}$ & $103,60 \mathrm{a}$ & $69,31 \mathrm{a}$ \\
BRS 8381 & $44,33 \mathrm{a}$ & $111,26 \mathrm{a}$ & $64,80 \mathrm{a}$ \\
\hline
\end{tabular}

As médias seguidas da mesma letra na coluna não diferem entre si, pelo teste de Tukey a 5\% de probabilidade. Fonte: Elaboração dos autores.

Na cidade de São José do Xingu-MT, a cultivar BRS 7380 apresentou uma produtividade de grãos de $68,31 \mathrm{sc} / \mathrm{ha}$, ou $4.098 \mathrm{~kg} / \mathrm{ha}$, maior que a produtividade encontrada por Libório et al. (2017) em experimentos com esta mesma variedade na cidade de GuaíraSP, onde obteve uma produtividade de $2.834 \mathrm{~kg} / \mathrm{ha}$ ou 47,2 sc/ha na safra 2016/2017.

A cultivar BRS 7780 IPRO atingiu neste experimento uma produtividade de 69,31 sc/ha ou 
$4.158 \mathrm{~kg} / \mathrm{ha}$, maior que a encontrada por Almeida et al. (2016) de 58,4 sacas/ha, também na cidade de Guaíra-SP, e relatou que sobre ataques e danos causados pela lagarta falsa medideira (Pseudoplusia includens), foi uma das cultivares com menor ataque desta lagarta sendo uma das mais produtivas, com $61 \%$ a mais do que as cultivares de pior desempenho, provavelmente devido sua tecnologia de resistência.

Segundo os dados apresentados nas Tabelas 3 e 5, tornou-se possível verificar que a cultivar BRS 8381 apresentou maior altura de inserção da primeira vagem, altura de planta na maturação e maior quantidade de vagem por planta, do que as encontradas por Gomes et al. (2017), no campo experimental da Embrapa Roraima, na cidade de Boa Vista-RR, que verificou uma altura de planta de $70,4 \mathrm{~cm}$; altura de inserção de primeira vagem $10,9 \mathrm{~cm}$; e número de vagens total 37.

A cultivar Pioneer 96Y90RR nesta região atingiu resultados superiores aos encontrados por Borges et al. (2018), no estado de São Paulo, cidade de Pindorama, que obteve para caracteres altura de primeira vagem $12 \mathrm{~cm}$, altura de plantas $63 \mathrm{~cm}$, e produtividade de $3.878 \mathrm{~kg} / \mathrm{ha}$ ou 64,63 sc/ha. Para essa cultivar ainda não existem dados literários registrados na região Araguaia-Xingu.

A cultivar Agro Norte 73017RR não apresenta dados na literatura por ser uma variedade que ainda se encontra em testes, os primeiros realizados foram na safra 2017/2018, e ainda será testada por mais uma safra antes do lançamento pela empresa Agro Norte Pesquisa e Sementes, sediada no município de Sinop-MT, os dados da mesma foram informados diretamente pela pesquisadora responsável Lorena Milani Matos Maronezzi. Outro teste desta variedade foi realizado pelos técnicos da empresa no município de Porto Alegre do Norte-MT, na Fazenda Santa Rita, onde foi obtida uma média de produtividade de $65 \mathrm{sc} / \mathrm{ha}$, o que assemelha-se com os resultados deste trabalho (Tabela 5).

Os dados obtidos através deste experimento para a cultivar Agro Norte 73017RR (Tabela 3) foram superiores aos aferidos pela pesquisadora Lorena M. M. Maronezzi na cidade de Sinop-MT, que obteve para altura de inserção de primeira vagem de apenas 4,7 cm e altura de planta na maturação de $86 \mathrm{~cm}$.

\section{CONCLUSÕES}

De forma geral as cultivares não se diferenciaram entre si e obtiveram resultados positivos, com exceção da BRS 7380RR que não apresentou resultado satisfatório conforme a média de produção atingida na região do experimento para safra 2017/2018.

Dentre as cultivares estudadas a BRS 7780IPRO apresentou média de produção de 
grãos de 69,31 sc/ha obtendo maior resultado, também se destacaram as cultivares Pioneer 96Y90RR com 68,21 sc/ha e BRS 7380RR com 68,31 sc/ha, podendo assim serem recomendadas para o cultivo nesta região.

Os resultados referentes a esta e demais cultivares utilizadas podem servir de referência para trabalhos futuros nesta região, auxiliando e proporcionando maior escolha pelos produtores no momento de definir a mais adaptada para sua realidade.

\section{REFERÊNCIAS}

AGRONORTE, Pesquisa e Sementes. Cultivar AN73017. Disponível em <http://www.agronorte.com.br/> acessado em 26 de junho de 2018.

ALMEIDA, R. E. et al. Desempenho de Cultivares de Soja na Região Centro Norte do Estado do Tocantins na safra 2015/2016. Embrapa Pesca e Aquicultura, Palmas,-TO, 2016.

BALBINOT JUNIOR, A. A. Contribuição dos ramos na produtividade da soja. Blog Embrapa Soja, 2018. Disponível em <https://blogs.canalrural.uol.com.br/embrapasoja/ 2018/05/22/contribuicao-dos-ramos-na-produtividade-da-soja/> acessado em 20 de julho de 2018.

BORÉM, A.; MIRANDA, G. V. Melhoramento de Plantas. 5. ed. Viçosa, MG: Ed. UFV, 2009, p 529.

BORGES, W. L. B. et.al. Avaliação regional de cultivares de soja no estado de São Paulo Safra 2017/18. $3^{\circ}$ ENCONTRO TÉCNICO SOBRE AS CULTURAS DA SOJA E DO MILHO NO NOROESTE PAULISTA. Nucleus, Edição Especial, 2018.

BRASIL. História da cidade de São José do Xingu. Disponível em $<$ http://www.camarasaojosedoxingu.mt.gov.br/o-munic\%C3\%ADpio/historia> acessado em 10 de julho de 2018.

CÂMARA, G.M.S. Soja: tecnologia da produção. Piracicaba, 1998.

CONAB. Companhia Nacional de Abastecimento. Acompanhamento da safra brasileira de grãos. V.1, n.1. Brasília, 2013.

CONAB. Companhia Nacional de Abastecimento. Acompanhamento da safra brasileira de grãos. V.5 Safra 2017/18 - N.7 Sétimo levantamento. Brasília, 2018. Disponível em <https://www.conab.gov.br/info-agro/safras/graos> acessado em 10 de junho de 2018.

DECICINO, T. A importância do posicionamento de cultivares de soja para o sucesso da cultura. InformaticoTécnicoano4,n.9.Technology Development, Monsanto. 2015.

DOMINGUES, M. S. Avaliação da monocultura de soja como matéria prima para a produção de biodiesel e sua relação com o desmatamento da floresta amazônica: estudo de caso na região de São José do Xingu (MT). Dissertação de mestrado programa de pôs 
graduação em energia, instituto de eletrotécnica e energia da universidade de são Paulo, 2010.

FERNÁNDEZ, A. J. C. Do cerrado à Amazônia: as estruturas sociais da economia da soja em Mato Grosso. 254 p. Tese (doutorado) - Universidade Federal do Rio Grande do Sul, 2007.

FERREIRA, D. F. Análise estatística por meio do SISVAR (Sistema para Análise de Variância) para Windows. Versão 4.0. In: REUNIÃO ANUAL DA REGIÃO BRASILEIRA DA SOCIEDADE INTERNACIONAL DA BIOMETRIA, 45. São Carlos:anais, 2000. 255-258 p.

FREITAS, M. C. M.; HAMAWAKI, O. T.; BUENO, M. R.; MARQUES, M. C. Época de semeadura e densidade populacional de linhagens de soja UFU de ciclo semi-tardio. Biosci. J., Uberlândia, v. 26, n. 5, p. 698-708, Sept./Oct. 2010.

FREITAS, M, C. M. A cultura da soja no brasil: o crescimento da produção brasileira e o surgimento de uma nova fronteira agrícola. Pós-graduando em Agronomia da Universidade Federal de Uberlândia, 2011.

FUNDAÇÃO, Cerrados (Brasil). Cultivares de soja. Disponível em <http://fundacaocerrados.com.br/> acessado em 24 de junho de 2018.

GARCIA, A.; PÍPOLO, A.E.; LOPES, I.O.N.; PORTUGAL, F.A.F. Instalação da lavoura de soja: época, cultivares, espaçamento e população de plantas. Londrina: Embrapa Soja, p.11. (Circular Técnica, 51), 2007.

GOMES, H. H. S. et al. Características agronômicas na produtividade da soja em diferentes densidades de plantas. XXX CONGRESSO BRASILEIRO DE AGRONOMIA. UFRR, Campus Monte Cristo, Boa Vista-RR, 2017.

HEIFFIG, L.S.; CÂMARA, G.M. de S. Soja: colheita e perdas. Piracicaba: ESALQ, 2006, p.37.

IBGE, (Brasil). Mapa de Solos do Estado de Mato Grosso. Instituto Brasileiro de Geografia e Estatistica. 2009. Disponível em <fftp://geoftp.ibge.gov.br/informacoes_ambientais/ pedologia/mapas/unidades_da_federacao/mt_pedologia.pdf $>$ acessado em 19 de julho de 2018.

LIBÓRIO, P. H. S. et.al. Desempenho produtivo de cultivares de soja em área de sequeiro Guaíra/SP. Safra 2016/17. Ciência \& Tecnologia: Fatec-JB, Jaboticabal, v. 9. Número especial, 2017.

PEIXOTO, C.P.; CÂMARA, G.M.S.; MARTINS, M.C.; MARCHIORI, L.F.S.; GUERZONI, R.A.; MATTIAZZI, P. Data de semeadura e densidade de plantas de soja: componentes de produção e rendimento de grãos. Scientia Agricola, Piracicaba, v.57, n.1, 2000, p.153-162.

PIMENTEL, G; GARCIA, C. H. Estatística aplicada a experimentos agronômicos e florestais: exposição com exemplos e orientações para uso de aplicativos. 1.ed. Piracicaba:FEALQ, 2002. 
PROCHNOW, L. I.; ROSSI, F. Análise de Solo e Recomendação de Calagem e Adubação. Viçosa, MG: CPT, 2009.

RAMOS, E. U. J. et al. Componentes de produção e produtividade de grãos de soja em função da densidade de plantas. Embrapa soja, Sinop- MT. 2017.

REZENDE, P.M.; CARVALHO, E.A. Avaliação de cultivares de soja (Glycine max (L.) Merrill) para o Sul de Minas Gerais. Ciência e Agrotecnologia, Lavras, v.31, n.6, 2007, p.1616-1623.

ROSSI. R. F. et al. Vigor de sementes, população de plantas e desempenho agronômico de soja. Revista Ciências Agrárias, v. 60, n. 3, 2017 p. 215-222.

SOJA, 96Y90. Cultivar tolerante ao Glifosato e ao Nematoide de Cisto da Soja. DuPont Pioneer. Disponível em <http://www.pioneersementes.com.br/soja/central-deprodutos/produtos/96y90> acessado em 4 de julho de 2018.

TORRES, F. E. et al. Desempenho de genótipos de soja nas condições edafoclimáticas do ecótono Cerrado Pantanal. Interações, Campo Grande, v. 15, 2014. p.71-78.

USDA, (Estados Unidos da América). World Agricultural Production. Unitet States Department of Agriculture. Foreign Agricultural Service. Circular Series WAP 7-18 July, 2018. Disponível em <https://apps.fas.usda.gov/psdonline/circulars/production.pdf > acessado em 20 de julho de 2018.

VALLE, L. C. S. et al. Diagnóstico de morte de pastagens nas regiões leste e nordeste do Estado de Mato Grosso. Campo Grande : Embrapa Gado de Corte, 2000, p.13. 